\title{
Preferred Phosphodiester Conformations in Nucleic Acids. A Virtual Bond Torsion Potential to Estimate Lone-Pair Interactions in a Phosphodiester
}

\author{
A. R. SRINIVASAN and N. YATHINDRA, Department of \\ Crystallography and Biophysics, University of Madras, Madras; and \\ V. S. R. RAO and S. PRAKASH, Molecular Biophysics Unit, Indian \\ Institute of Science, Bangalore, India
}

\begin{abstract}
Synopsis
The lone-pair orbital interactions arising in a phosphodiester are incorporated into semiempirical conformational energy calculations using a unifold "torsional potential" around the virtual bond linking the ester oxygen atoms. The results explain the observed experimental data better than other methods.
\end{abstract}

\section{INTRODUCTION}

The preferred orientations around the internucleotide P-O $\left(3^{\prime}\right)\left(\omega^{\prime}\right)$ and P-O $(5)(\omega)$ bonds of the phosphodiester which link the successive nucleotide residues are crucial in discerning the helical as well as nonhelical bend conformations of nucleic acids and polynucleotides. Experimental investigations other than the single-crystal $x$-ray diffraction methods are unable to provide directly information about $\mathrm{P}-\mathrm{O}$ bond conformations, except possibly for the stacked conformation, although the results of these studies have also conclusively substantiated the predominance of the preferred nucleotide conformations in di- and oligonucleotides. Calculations using semiempirical potential functions ${ }^{1-3}$ and semiempirical quantum mechanical methods ${ }^{4,5}$ on model sugar-phosphate-sugar backbone segments have aided in delineating the stereochemically possible and the preferred conformations around the $\mathrm{P}-\mathrm{O}$ bonds in di-, oligo-, and polynucleotides. However, some of these remained less satisfactory to the extent that a low-energy minimum occurred corresponding to the fully extended phosphodiester conformation $\left(\omega^{\prime}, \omega\right)\left(180^{\circ}, 180^{\circ}\right)(t t)$, which was argued $^{6}$ to be a considerably high energy conformation owing to the repulsive interactions between the lone-pair orbitals associated with the backbone ester oxygen $\mathrm{O}\left(5^{\prime}\right)$ and $\mathrm{O}\left(3^{\prime}\right)$ atoms. Also, no such conformation has been experimentally observed. Recent quantum chemical calculations ${ }^{7-9}$ have shown that the fully extended phosphodiester conformation $(t t)$ is indeed energetically unfavorable, in agreement with the earlier 
chemical reasonings of Sundaralingam ${ }^{6}$ and with the estimated repulsive interaction energy due to eclipsed lone-pair interactions (which are of the order of $4-7 \mathrm{kcal} / \mathrm{mol}$ ). It is important that this aspect be introduced into the semiempirical potential functions to enhance their predictive abilities. A straightforward way of rendering the $(t t)$ conformation a high-energy one is to introduce a twofold torsion potential around both the ester $\mathrm{P}-\mathrm{O}$ bonds with a barrier height equivalent to half the repulsive lone-pair interaction energy. ${ }^{10}$ The use of such torsional potentials around the $\mathrm{P}-\mathrm{O}$ bonds renders the extended $(t t)$ as a high-energy conformation due to the very nature of the potential function; however, this fails to convey the physical picture of the directional dependence of the lone-pair interaction.

We propose here a potential function which avoids the use of an unrealistically high torsion potential around the $\mathrm{P}-\mathrm{O}$ bonds but directly estimates the lone-pair interaction energy in a phosphodiester bond in relation to directional properties of the bonds. The interactions between the lone-pair orbitals in a phosphodiester is regarded as arising from a "torsion potential" around the virtual bond linking the ester $\mathrm{O5}^{\prime}$ and $\mathrm{O}^{\prime}$ oxygen atoms (Fig. 1), akin to the torsion potential around the chemical bonds. The lone-pair interaction potential is expressed as $V_{A n}=$ $V a / 2(1+\cos \theta)$, where $\theta$ is the dihedral angle about the virtual bond joining the two ester oxygen atoms (Fig. 1 ). The torsion angle $\theta$ is taken as zero for the eclipsed orientations of the lone pairs. The directions $\mathrm{O}\left(3^{\prime}\right)$-A and $\mathrm{O}\left(5^{\prime}\right)$-B in Fig. 1 represent the perpendicular bisectors of the two lone pairs. The characteristics of this potential are such that the energy is maximum for the phosphodiester conformation $\left(\omega^{\prime}, \omega\right)=\left(180^{\circ}, 180^{\circ}\right)$ at which the two lone-pair orbitals are eclipsed or in a cis conformation, i.e., $\theta=0^{\circ}$, and the lone-pair interaction energy is minimum for phosphodiester conformations $\left(\omega^{\prime}, \omega\right)=\left(0^{\circ}, 180^{\circ}\right),\left(180^{\circ}, 0^{\circ}\right),\left(60^{\circ}, 60^{\circ}\right)$, and $\left(300^{\circ}, 300^{\circ}\right)$, when the lone-pair orbitals are in a trans conformation, i.e., $\theta=180^{\circ}$. This is equivalent to a singlefold torsional potential around the virtual bond, and a simple correlation between $\theta$ and $\omega^{\prime}$ and $\omega$ does not exist. The value of $2.8 \mathrm{kcal} / \mathrm{mol}$

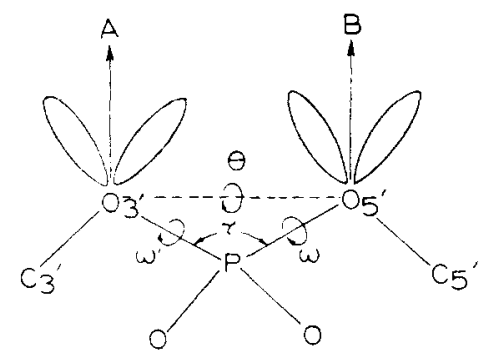

Fig. 1. Schematic representation of lone-pair orbitals associated with $O\left(3^{\prime}\right)$ and $O\left(5^{\prime}\right)$ ester oxygen atoms in a phosphodiester. The dotted line joining the $O\left(3^{\prime}\right)$ and $O\left(5^{\prime}\right)$ represents the virtual bond and the directions $\mathrm{O}\left(3^{\prime}\right)-\mathrm{A}$ and $\mathrm{O}\left(5^{\prime}\right)-\mathrm{B}$ are the directions of the lone-pair axes. $\theta$ defines the torsion angle around the virtual bond $\mathrm{O}\left(3^{\prime}\right)-\mathrm{O}\left(5^{\prime}\right)$ and is defined as zero for the cis orientation of the $\mathrm{O}\left(3^{\prime}\right)$-A and $\mathrm{O}\left(5^{\prime}\right)$-B bonds. 
has been assigned for $V a$ from studies ${ }^{11}$ of anomeric effect in sugars. This "anomeric torsional potential" around the virtual bond affords a direct visualization of the correlation of the lone-pair orbital orientations with their energies while at the same time avoiding the necessity of using twofold torsion potentials around each of the $\mathrm{P}-\mathrm{O}$ bonds. Further, we find that a threefold torsion potential with the usual low barrier height of $1 \mathrm{kcal} / \mathrm{mol}$ is adequate to explain the experimental observations. The use of this anomeric potential in conjunction with the conventional semiempirical potential functions produces better agreement between theoretical predictions and experimental observations. Similar potential functions used for estimating the lone-pair interactions in polysaccharides ${ }^{11}$ have resulted in remarkable agreement between the theoretically computed and experimentally observed unperturbed end-to-end dimensions.

\section{METHOD}

The total potential energy in a ribodinucleoside monophosphate and ribodinucleoside triphosphate sugar-phosphate-sugar backbone is computed as a function of the internucleotide $\mathrm{P}-\mathrm{O}\left(3^{\prime}\right)\left(\omega^{\prime}\right)$ and $\mathrm{P}-\mathrm{O}\left(5^{\prime}\right)(\omega)$ torsions, using the semiempirical potential functions and also the above anomeric torsional potential around the virtual bond (which joins the ester oxygen atoms to take into account the lone-pair interactions). The interactions due to the bases are excluded with the intention of probing the inherent.property characteristics of the polynucleotide backbone. The potential functions and the constants used here are the same as those adopted earlier. ${ }^{3}$ The $\left(\omega^{\prime}, \omega\right)$ energy surfaces are shown in Figs. 2 and 3.

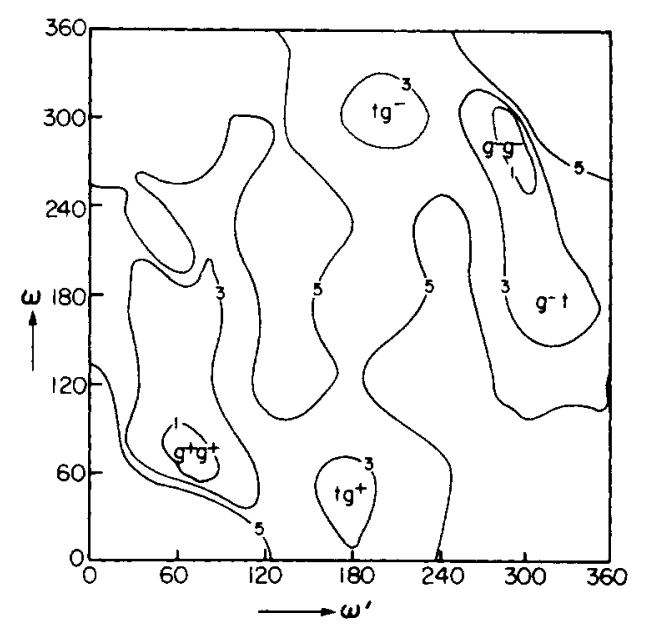

Fig. 2. The $\left(\omega^{\prime}, \omega\right)$ conformational energy map obtained for a dinucleoside monophosphate with the $\mathrm{C}\left(3^{\prime}\right)$-endo geometry for the nucleotides. The lone-pair orbital interaction energies are included by the use of virtual bond torsion potential. A threefold torsion potential around the $\mathrm{P}-\mathrm{O}$ bonds with a barrier height of $1 \mathrm{kcal} / \mathrm{mol}$ is also included. 


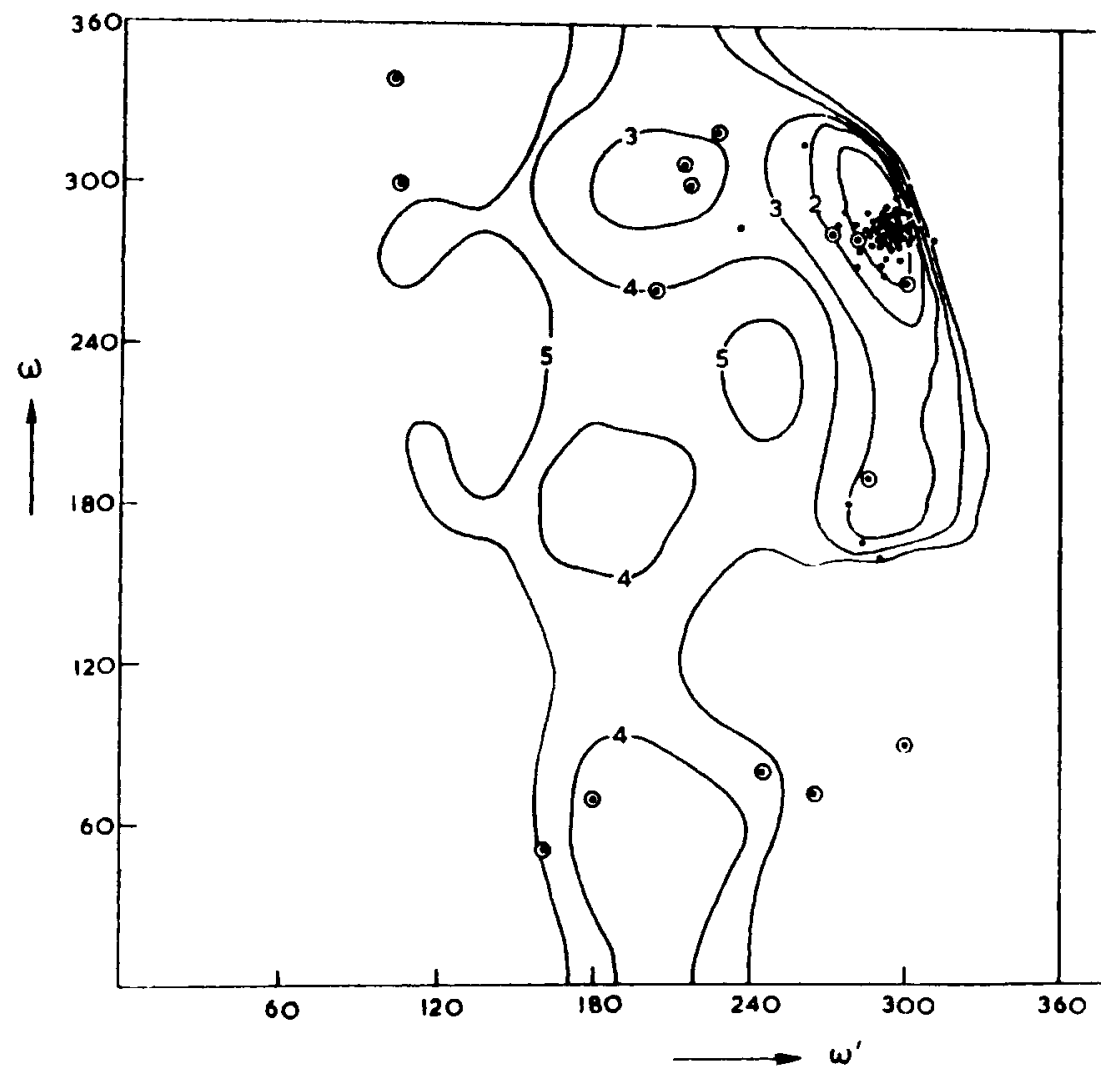

Fig. 3. The $\left(\omega^{\prime}, \omega\right)$ conformational energy map obtained for a dinucleoside triphosphate with $\mathrm{C}\left(3^{\prime}\right)$-endo geometry for the nucleotides. The lone-pair orbital interaction energies are included by the use of the virtual bond torsion potential. A threefold torsion potential around the $\mathrm{P}-\mathrm{O}$ bonds with a barrier height of $1 \mathrm{kcal} / \mathrm{mol}$ is also included.

\section{RESULTS AND DISCUSSION}

Figure 2 shows the $\left(\omega^{\prime}, \omega\right)$ energy map obtained for a $\mathrm{C}\left(3^{\prime}\right)$-endo ribonucleoside monophosphate backbone that also takes into account the lone-pair interaction energy through a virtual bond torsion potential of barrier height equal to $2.8 \mathrm{kcal} / \mathrm{mol}$. The most important feature in the map is that contrary to earlier results obtained using semiempirical potential functions $^{1-3}$ and some earlier molecular orbital computations ${ }^{4,5}$ but in agreement with the refined calculations of this type, ${ }^{7-9}$ the fully extended $t t$ conformation is now rendered a considerably high energy conformation. Similarly, the phosphodiester conformation $g^{-} t=\left(\omega^{\prime}, \omega\right)=\left(300^{\circ}, 180^{\circ}\right)$, $g^{+} t\left(\omega^{\prime}, \omega\right)=\left(60^{\circ}, 180^{\circ}\right)$ is about $2 \mathrm{kcal} / \mathrm{mol}$ higher in energy than the most stable $g^{+} g^{+}$and $g^{-} g^{-}$phosphodiesters. The $t g^{-}=\left(180^{\circ}, 300^{\circ}\right)$ and the $\operatorname{tg}^{+}\left(180^{\circ}, 60^{\circ}\right)$ phosphodiesters are about $2.5 \mathrm{kcal} / \mathrm{mol}$ higher in energy than the global minimum found earlier. Except for one of the molecules of UpA, ${ }^{12,13}$ the rest of the crystal structures of ribodinucleoside monophosphates favor either the $g^{-} g^{-}$or the $g^{+} g^{+}$phosphodiesters. 
Similarly, the $\left(\omega^{\prime}, \omega\right)$ conformational energy map (Fig. 3) obtained for a dinucleoside triphosphate backbone, which is a better representation of a polynucleotide backbone chain, also shows that the fully extended $t t$ phosphodiester conformation is now rendered energetically highly unfavorable. This indicates that the explicit inclusion of lone-pair orbital interactions through a "torsion potential" around the virtual bond satisfactorily accounts for the high energy associated with the fully extended phosphodiester conformation, in conformity with experimental observations. ${ }^{6}$ The global minimum in Fig. 3 occurs at $\left(\omega^{\prime}, \omega\right) \simeq\left(300^{\circ}, 300^{\circ}\right)$ and is now stable over the other stereochemically possible $g^{-} t, \operatorname{tg}^{-}$, and $t g^{+}$ conformations by about $2.5 \mathrm{kcal} / \mathrm{mol}$. Values greater than $2.8 \mathrm{kcal} / \mathrm{mol}$ for the barrier height do not alter any of the above conclusions except that the energies associated with the $t t, t g^{+}, t g^{-}$, and $g^{-} t$ conformations become correspondingly higher than the stable $g^{-} g^{-}$conformation.

We find that when only the twofold torsion potentials around the chemical $\mathrm{P}-\mathrm{O}$ bonds are used (without the threefold potential), two more minima become important in the $\left(\omega^{\prime}, \omega\right)$ map (not shown) for ribodinucleoside monophosphate: one around $\left(\omega^{\prime}, \omega\right) \simeq\left(90^{\circ}, 270^{\circ}\right)$ having an energy equivalent to $g^{+} g^{+}$and $g^{-} g^{-}$and another around $\left(\omega^{\prime}, \omega\right) \simeq$ $\left(300^{\circ}, 120^{\circ}\right)$, which is about $2 \mathrm{kcal} / \mathrm{mol}$ higher than the global minimum. Only the minimum around $\left(90^{\circ}, 270^{\circ}\right)$ becomes important for ribodinucleoside triphosphate. However, inclusion of a threefold torsion potential with an unrealistically high barrier height of $3 \mathrm{kcal} / \mathrm{mol}$ readily renders these minima high-energy conformations because of the nature of the $\left(\omega^{\prime}, \omega\right)$ values at which the minima occur and because of the torsion potential. A low barrier height of the order of $1 \mathrm{kcal} / \mathrm{mol}$ is found to be insufficient to make them energetically less important. Comparison of the shape and the general features of the $\left(\omega^{\prime}, \omega\right)$ map obtained here (Fig. 3) with the $\left(\omega^{\prime}, \omega\right)$ map of Ref. 10 suggests that the use of twofold torsional potential around the chemical $\mathrm{P}-\mathrm{O}$ bonds considerably restricts their rotational freedom.

A comparison of the above results with the phosphodiester conformations found ${ }^{14-17}$ in the crystal structures of the two polymorphs of yeast tRNA ${ }^{\text {Phe }}$ would provide a measure of agreement between theory and experiment. While there are only minor differences in the conformational angles, probably due to alternative interpretation and degree of refinement, the overall conformations in these structures are very similar. Hence phosphodiester conformations found in the monoclinic form ${ }^{14,15}$ alone are plotted in Fig. 3. The dots represent phosphodiesters which are flanked on either side by the $\mathrm{C}\left(3^{\prime}\right)$-endo nucleotide geometry with the approximate gauche ${ }^{+}\left(60^{\circ}\right)$ conformation around the $\mathrm{C}\left(4^{\prime}\right)-\mathrm{C}\left(5^{\prime}\right)$ bond. The dots enclosed in the circles correspond to the phosphodiesters flanked at least on one side by a nucleotide having a trans $\left(180^{\circ}\right)$ or a gauche- $\left(300^{\circ}\right)$ geometry around the $\mathrm{C}\left(4^{\prime}\right)-\mathrm{C}\left(5^{\prime}\right)$ bond or a $\mathrm{C}\left(2^{\prime}\right)$-endo pucker or both. It is noteworthy that none of the phosphodiesters occur in the $t t$ region forbidden by lone-pair repulsive interactions, and a large number of points cluster around the most favored $g^{-} g^{-}$domain. The three dots which correspond 
to phosphodiesters in the bend regions lie in the high-energy $g^{-} t$ domain. Thus all the points lie within the boundary prescribed by the $5 \mathrm{kcal} / \mathrm{mol}$ energy contour. Other points, some of which lie outside the energy contours, should be compared with the $\left(\omega^{\prime}, \omega\right)$ maps obtained with appropriate geometry for the nucleotides.

\section{CONCLUSIONS}

A unifold torsional potential around the virtual bond linking the ester oxygen atoms in a phosphodiester bond is suggested to account for the lone-pair orbital interactions in semiempirical calculations. Consideration of this virtual bond torsion potential along with the conventional potential functions satisfactorily accounts for the high energy associated with the fully extended $t t$ phosphodiester conformation in nucleic acids and polynucleotides and also the observed experimental data. The important feature of this approach is that it affords a clear visualization of the correlation between the energetics and the orientations of the lone-pair orbitals. The use of the simple "virtual bond torsion potential" to account for the lone-pair interactions in a phosphodiester bond should also be useful in the analysis of conformations of coenzymes like NAD, FAD, nucleoside diand triphosphates, and phosphoglycerides. An estimate of the lone-pair interactions arising as a result of conformational and configurational changes in nucleosides could also be obtained by the use of such virtual bond torsion potentials.

Contribution No. 494 from the Department of Crystallography and Biophysies and Contribution No. 138 from the Molecular Biophysics Unit, Indian Institute of Science, Bangalore.

\section{References}

1. Sasisekharan, A. V. \& Lakshminarayanan, A. V. (1969) Biopolymers 8, 505-514.

2. Olson, W. K. \& Flory, P. J. (1972) Biopolymers 11, 25-26.

3. Yathindra, N. \& Sundaralingam, M. (1974) Proc. Natl. Acad. Sci. USA 71, 33253328.

4. Saran, A. \& Govil, G. (1971) J. Theor. Biol. 33, 407-418.

5. Pullman, B., Perahia, D. \& Saran, A. (1972) Biochim. Biophys. Acta 269, 1-14.

6. Sundaralingam, M. (1969) Biopolymers 7, 821-869.

7. Newton, M. (1973) J. Am. Chem. Soc. 95, 256-258.

8. Perahia, D., Pullman, B. \& Saran, A. (1973) C. R. Acad. Sci, 277, 2257-2260.

9. Perahia, D., Pullman, B., Vasilescu, D., Cornillon, R. \& Broch, H. (1977) Biochim. Biophys. Acta 478, 244-258.

10. Govil, G. (1976) Biopolymers 15, 2303-2307.

11. Prakash, S., Srinivasan, A. R. \& Rao, V. S. R. (1978) in Collected Abstracts, Proceedings of the International Symposium on Biomolecular Structure, Conformation, Function and Evolution, January 4-7, 1978, Madras, India. 
12. Rubin, J., Brennan, T. \& Sundaralingam, M. (1972) Biochemistry 11, 3112-3127.

13. Sussman, J. L., Seeman, N. C., Kim, S. H. \& Berman, H. M. (1972) J. Mol. Biol. 66, $403-421$.

14. Jack, A., Ladner, J. E. \& Klug, A. (1976) J. Mol. Biol. 108, 619-650.

15. Stout, C. D., Mizuno, H., Rubin, T., Brennan, T., Rao, S. T. \& Sundaralingam, M. (1976) Nucleic Acids Res. 3, 1111-1123.

16. Quigley, G. J., Seeman, N. C., Wang, A. H. J., Suddath, F. L. \& Rich, A. (1975) Nucleic Acids Res. 2, 2329-2334.

17. Sussman, J. L. \& Kim, S. H. (1976) Biochem. Biophys Res. Commun. 68, 89-96.

Received November 21, 1978

Accepted July 20, 1979 